\title{
Ba Duan Jin Teaching Process Should Pay Attention to the Problem
}

\author{
Bao-Hao SONG \\ Nan Yang Medical College
}

Keywords: Ba Duan Jin, Teaching, Attention to the problem.

\begin{abstract}
Baduanjin belong Qigong is ancient China's guided surgery, has been widely circulated in the country, a health effect is very obvious, I found in teaching, some students in the practice Ba Duan Jin process, there is insufficient preparation, improper exercise, relaxation activities mere formality, uncoordinated movements and breathing problems. This paper tries to elaborate on these issues, for the students and the community to help.
\end{abstract}

\section{Foreword}

The exercises have the following characteristics:1. The combination of fitness and disease prevention. Health effects have been recognized and accepted by the masses, good physical exercise, the body's resistance will be enhanced, they will naturally play a role in disease prevention, namely Chinese medicine talked about "righteousness deposit, evil can not do"; 2. The combination of movement and breathing. Breathing has renewal effect, that is, more oxygen inhaled fresh air, discharge polluted gas in vivo, thereby promoting blood to run, and exercise can also promote blood meridian running, coordination and cooperation between the two can better promote fitness action; 3. movement and ideas combined. Combined with exercise and ideas that can move people outside and inside the quiet, so external movement phase and limb movement; 4. pictogram combination with limb movement. The set of fitness exercises certain action or from life, or imitate animal movements, such as "chasing its tail" can achieve the removal of "Firelight" purposes, "footed" can play a role chest, lung declared down the.

This action ancients likened to "Kam", meaning action stretch, like brocade as beautiful, supple, and because the exercises for a total of eight, each action, named for the "Ba Duan Jin." Ba Duan Jin, "eight" the word, not a single finger segments, sections, and eight action, but rather that such gossip as it exercises a variety of elements, mutual restraint, mutual contact, cycle operation. "Jin" word can also be understood as a collection of individual guided surgical procedures, such as continuous silk brocade, it is a complete health and fitness. The entire exercise gentle stretching, profits slip smoothly; pine have tight movement phase and; air-smooth, soft bone positive reinforcement.

\section{Current Status and Problems}

Now we practice fitness exercises - Ba Duan Jin, the State Sports General Administration Health Qigong Management Center in 2002 organized experts and scholars to compose and officially launched in 2003, a set of Qigong. Adapted after this power law, whether it is exercise intensity or ordering of the action, are more in line with the law of kinesiology and physiology, it is a secure and reliable small-scale aerobic exercise. It is based on traditional Chinese philosophy as a guide, combined with Chinese medicine theory, the use of the guide, means qi to improve the masses health and quality of life as a fitness exercises purposes. Practitioners unrestricted age, gender, site conditions, the weather is good, the place in the outdoor Kongkuo practitioners encounter wind, rain, snow, dust storms and other bad weather, you can choose the indoor practice practice, as long as there is space 4 square meters, can practitioners, and does not require any equipment, easy to carry in the masses, however, I found that in the teaching, whether it is the learning process, or the masses practitioners Baduanjin process, there are the following problems. 


\section{Insufficient Preparation}

Exercise Baduanjin do some preparatory activities before it is necessary, as former extreme sports need to do some joint activities, and other warm-up exercises, like jogging, before practicing Baduanjin have to do what the best preparations for active blood, activate fine gas. Preparations well, the essence of the blood in an active state of excitement, practitioners will be more effective, more conducive to play the energy of the body. Commonly used methods are: standing natural relax, mix thoroughly breathing, followed by doing circling motion waist, shoulders, neck and knee, can promote blood from the body's largest joint center "waist" started its activities diverge up activities shoulders, neck, knee down activities, ankle, so blood will center around several active start automatically opened. I feel from years of practicing the pranayama relax from a standing start good, but with all the major joints circling activity, easy access to practice state of flux. Preparatory activities before practice includes two aspects: physical preparation, psychological preparation. Class conventional practice is to let my students about the standard running track, the weather is cold run 2-3 laps, but also ran a lap warmer weather, which for most students, it is not a problem, but there are a few Students do not want to run, that is the excess, this idea is wrong. Then the body activities, especially the shoulder, waist, knee, ankle joint activities to the full, only the body's muscles, joints fully, before entering the practice stage; mental preparation include breathing evenly, muscle aches may occur, fatigue and other phenomena, there must be adequate psychological preparation. If not fully prepared, that's not a workout, but the destruction of the body, often caused by sports injuries, the result will be counterproductive.

\section{Physical Activity and Exercise Intensity Inappropriate}

Baduanjin The fitness exercises for young students and middle-aged people, the moderate-intensity physical activity and exercise, and for the elderly or those who suffer from diseases, physical activity and exercise intensity may appear bigger. Measure the amount and size of exercise intensity, there is a standard is the "target heart rate", the formula is: target heart rate $=(220$ - age $) \times 65 \%-85 \%$, is generally believed to be less than the target heart rate HR practice, but because of the existence individual differences, target heart rate as a reference only. Mastery of the exercise should be subject to proprioception, the most simple and effective method of detection is a happy after exercise, pulse steady, normal blood pressure, loss of appetite and sleep well, indicating that the amount of exercise is appropriate. If the body was tired after exercise, the pulse time is not restored, loss of appetite, poor sleep, then the excessive movement should be timely adjusted. The size and intensity of exercise, the following three cases: 1 . If the practice a Weihan, subjective feeling of general air-smooth, appetite, sleep better, the next day energetic, did not feel fatigue, it shows the amount of exercise and moderate exercise intensity, should insist on this exercise volume and intensity; 2. If after practice excessive sweating, causing palpitation, shortness of breath, dizziness, loss of appetite, sleep well, the next day malaise, do not want to move, indicating that physical activity and exercise intensity is too large, the need to reduce the amount of exercise and exercise intensity; 3. If there is no practice after slightly sweated, and heart rate, breathing and did not change much compared to practice, indicating physical activity and exercise intensity is too small, you need to appropriately increase the amount of exercise and exercise intensity. Of course, the above three cases is not absolute, and to make concrete analysis of specific situations. 


\section{Movement and Breathing, Uncoordinated Ideas}

Qigong, the ancient called "guided surgery", namely "command and air conductivity, chin so soft", that is, through body movement, with the breath, ideas and other means to make the air-gentle smooth, soft round living body. Baduanjin The fitness exercises in addition to ready position and income potential, there are eight action, they are: hands care heaven triple burner, footed like Condor, spleen and stomach to be a single move, look back five workers and injured seven, Yaotoubaiwei go Firelight, hands climb back foot Gushen, save boxing increase strength angry, behind Britain riddled with seven consumer. In addition to hands climb back foot Gushen should shun abdominal breathing, the remaining paragraphs should use reverse abdominal breathing. The so-called cis abdominal breathing is to inhale projecting lower abdomen, abdominal contractions, abdominal contractions when breathing, abdominal bulge; the reverse abdominal breathing abdominal contraction inhale, protruding upper abdomen, lower abdomen bulge exhale, abdominal contraction. The specific method is to reverse abdominal breathing, inspiratory anus, abdomen, diaphragm rises. Exhale the diaphragm down, abdominal pine, pine anus. Follow When combined with the action from the suction off the call, open pull call, accumulator suction breathing principles made the call, and the tightness in each paragraph of the main action and movement changes alternately at the use of breath as "hands care heaven triple burner," a style, hands on Tropsch, inhale; while maintaining thrust, breath; when hands whereabouts of breath. In novice action, to give priority to natural breathing, do not deliberately pursue breathing fine, uniform, deep, long, do not deliberately pursue with the breath and movement, do not let the breathing psychological burden, to avoid dizziness, nausea, palpitation, shortness of breath and so on. To vary, capabilities, movement and breathing in keeping with the flow, do not tune into the state and self-adjusting in the progressive. The method is to use nose breathing inhale and exhale when breathing should be soft, thin, slow and long. Novice to natural breathing as well, until after the operation gradually skilled exercises can be combined with action based on breathing, breathing should be soft and uniform, not the pursuit of deep, natural breathing during exercise is indispensable, it plays an important regulatory role. Thus, over a longer period of time practicing, breathing and movements to meet freely, and gradually into disharmony and self-adjusting state.

\section{Relaxation Activities Formality}

After practicing relaxation activity is also very important aspect, as were tapping massage to relax, alternating upper and lower limbs shake relax, or do some simple aerobics slow. If conditions permit, before going to bed at night with warm water bubbles feet, appropriate to do some massage to promote blood circulation, so as to achieve the elimination of fatigue. After a workout during the Weihan, underwear should be replaced to prevent the common cold. After the practice, do not stop exercising immediately, to make the body gradually restored to the quiet state.

\section{References}

[1] Crist CM et al. Physical exercise increase natural cellularmediated tumor cytoxicity in elderly women [J]. Geronotol, 1989, 35:66. (In Chinese)

[2] Witt C, Becker M, Bandelin K, et al. Qigong for schoolchildren: A pilot study [J]. J Altern Complement Med, 2005 (11): 42-26. (In Chinese)

[3] Lee M-S, LimH-J, LeeMS. Impact of qigong exercise on self efficacy and other cognitive perceptual variables in patients with essential hypert.

[4] Barnes VA, Bauza LB, Treiber FA.Impact of st ress reduction on negat ive school behavior in adolescents [ J] .Health Qual Lif e Outcomes , 2003(1):1 -10. (In Chinese) 
[5] Wit t C , Becker M , Bandelin K , et al.Qigong for schoolchildren :Apilot study [ J] .J Altern Complement Med , 2005(11):41 -47. (In Chinese) 